\title{
TWO APPROACHES TO SUPERMANIFOLDS
}

BY

\author{
MARJORIE BATCHELOR
}

\begin{abstract}
The problem of supplying an analogue of a manifold whose sheaf of functions contains anticommuting elements has been approached in two ways. Either one extends the sheaf of functions formally, as in the category of graded manifolds [3], [8], or one mimicks the usual definition of a manifold, having replaced Euclidean space with a suitable product of the odd and even parts of an exterior algebra as in the category of supermanifolds [6]. This paper establishes the equivalence of the category of supermanifolds with the category of graded manifolds.
\end{abstract}

Introduction. Supermanifolds or graded manifolds were defined to provide a "space" whose "functions" would include anticommuting elements. There have been two approaches to the definition of these objects, emerging from two approaches to the study of the geometry of a manifold. Traditionally, the differential geometer regards the space itself as the primary object, but it is also possible to take the algebraic geometer's point of view and study the geometry of the space through the algebraic structure of its sheaf of functions. Supermanifolds as defined by de Witt [6] for example, follows the first approach, while graded manifolds, as defined by Kostant [8] are inspired by the algebraic geometer's approach. The purpose of this paper is to establish the equivalence of the category of supermanifolds with the category of graded manifolds.

In the first section, basic definitions are given and the main theorem is stated. Smooth maps on "super-Euclidean space" are constructed in $\$ 2$, and the proof of the equivalence theorem is given in $\$ 3$.

I would like to thank Professor S. Sternberg for bringing de Witt's work to my attention, and for subsequent discussions, and also Carolyn Schroeder for her help in finding the "right" definition for smooth maps on super-Euclidean space.

1. Definitions and results. All algebras and vector spaces are over the real numbers, although similar constructions could be carried out using complex numbers. All (ordinary) manifolds are considered to be real, smooth, Hausdorff paracompact manifolds.

1.1. Definition. An algebra $A$ is called a $\mathbf{Z}_{2}$-graded algebra (or simply a graded algebra) if $A$ can be written as a direct sum of linear subspaces $A=A_{0} \oplus A_{1}$ such that

$$
A_{i} A_{j} \subset A_{i+j(\bmod 2)}
$$

Received by the editors March 28, 1979.

AMS (MOS) subject classifications (1970). Primary 58A05.

Key words and phrases. Supermanifolds, graded manifolds, graded Lie algebras, superspace, anticommuting coordinates. 
An element $a$ in $A_{i}$ is said to be homogeneous of degree $i$. Let $|a|$ denote the degree of a homogeneous element $a$. The algebra $A$ is said to be graded commutative if $a b=(-1)^{|a||b|} b a$.

1.2. ExAmples. (1) Let $V$ be a vector space and suppose that $V$ is given a decomposition into two linear subspaces, $V=V_{0} \oplus V_{1}$. Then $\operatorname{End}(V)$ has the structure of a graded algebra setting $\operatorname{End}(V)=(\text { End } V)_{0} \oplus(\text { End } V)_{1}$ where

$$
(\operatorname{End}(V))_{i}=\left\{\delta \in \operatorname{End}(V): S V_{j} \subset V_{i+j(\bmod 2)}\right\}
$$

(2) Let $\Lambda \mathbf{R}^{s}$ denote the exterior algebra on $s$-dimensional real space. Then $\Lambda \mathbf{R}^{s}$ has the structure of a graded commutative algebra, setting $\Lambda \mathbf{R}^{s}=\left(\Lambda \mathbf{R}^{s}\right)_{0} \oplus\left(\Lambda \mathbf{R}^{s}\right)_{1}$ where $\left(\Lambda \mathbf{R}^{s}\right)_{0}$ is the subspace spanned by elements of even exterior degree, and $\left(\Lambda \mathbf{R}^{s}\right)_{1}$ is the subspace spanned by elements of odd exterior degree.

1.3. Definition. A graded manifold is a pair $(X, A)$ where $X$ is an ordinary manifold, called the underlying manifold of $(X, A)$, and $A$ is a sheaf of $\mathbf{Z}_{2}$-graded commutative algebras over $X$ which satisfies the following conditions

(i) There exists a surjective map of sheaves of graded algebras

$$
\theta: A \rightarrow C^{\infty}
$$

where $C^{\infty}$ is the sheaf of smooth functions on $X$ equipped with the grading $\left(C^{\infty}(V)\right)_{0}=C^{\infty}(V)$ for any open set $V$ in $X$.

(ii) Local triviality condition. There exists an open cover $\left\{V_{i}\right\}$ of $X$ and isomorphisms $T_{i}$ of graded algebras

$$
T_{i}:\left.\left.A\right|_{V_{i}} \rightarrow C^{\infty} \otimes \Lambda \mathbf{R}^{s}\right|_{V_{i}}
$$

where the sheaves $\left.A\right|_{V_{i}}$ and $\left.C^{\infty} \in \Lambda \mathbf{R}^{s}\right|_{V_{i}}$ are the sheaves $A$ and $C^{\infty} \otimes \Lambda \mathbf{R}^{s}$ restricted to the open set $V_{i}$, and where the grading on $C^{\infty} \otimes \Lambda \mathbf{R}^{s}$ is determined by $\left(C^{\infty}(V) \otimes \Lambda \mathbf{R}^{s}\right)_{0}=C^{\infty}(V) \otimes\left(\Lambda \mathbf{R}^{s}\right)_{0}$ for any open set $V$ in $X$. The integer $s$ is called the odd-dimension of $(X, A)$.

A morphism of graded manifolds from $(X, A)$ to another graded manifold $\left(X^{\prime}, A^{\prime}\right)$ is an algebra homomorphism

$$
\sigma: A^{\prime}\left(X^{\prime}\right) \rightarrow A(X)
$$

Let $\mathcal{G} \mathfrak{R}_{L}$ denote the category of graded manifolds, with odd dimension $s<L$ for some fixed integer $L$.

1.4. ExAmples. (1) The pair $\left(X, C^{\infty}\right)$ is a graded manifold. If $B_{s}$ is the sheaf over a point ${ }^{*}$ determined by $B_{s}\left(^{*}\right)=\Lambda \mathbf{R}^{s}$, then $\left({ }^{*}, B_{s}\right)$ is a graded manifold.

(2) The pair $(X, \Omega)$ is a graded manifold where $\Omega$ is the sheaf of smooth forms on $X$. More generally, if $E \rightarrow X$ is any finite dimensional vector bundle over $X$, one can form $\Lambda E \rightarrow X$, the exterior bundle associated with $E$. If $\Gamma(, \Lambda E)$ denotes the sheaf of sections of $\Lambda E$, then $(X, \Gamma(, \Lambda E))$ is a graded manifold. In fact, if $(X, A)$ is any graded manifold, there exists a vector bundle $E$ such that $(X, A)$ is isomorphic to $(X, \Gamma(, \Lambda E))$. The proof of this is given in [1].

Supermanifolds are defined by mimicking the usual definition of a manifold, replacing the real numbers by a large exterior algebra. Except for a difference in the definition of "smooth maps", the definition is essentially that given by de Witt [6]. 
1.5. Definition. Let $L, r, s$ be integers and assume that $L>s$. Define $(r, s)$-dimensional super-Euclidean space, $E^{r, s}$, to be the product of $r$ copies of $\left(\Lambda \mathbf{R}^{L}\right)_{0}$ with $s$ copies of $\left(\Lambda \mathbf{R}^{L}\right)_{1}$, i.e.

$$
E^{r, s}=\left(\Lambda \mathbf{R}^{L}\right)_{0}^{r} \oplus\left(\Lambda \mathbf{R}^{L}\right)_{1}^{s} .
$$

The topology on $E^{r, s}$ is defined in the following manner.

The augmentation map

$$
\varepsilon: \Lambda \mathbf{R}^{L} \rightarrow \mathbf{R}
$$

which assigns $u$ in $\Lambda \mathbf{R}^{L}$ to its component of exterior degree 0 gives rise to a map which will also be denoted by $\varepsilon$,

$$
\varepsilon: E^{r, s} \rightarrow \mathbf{R}^{r}
$$

given by $\varepsilon\left(u_{1}, \ldots, u_{r}, v_{1}, \ldots, v_{s}\right)=\left(\varepsilon\left(u_{1}\right), \ldots, \varepsilon\left(u_{r}\right)\right)$. A set $U$ in $E^{r, s}$ is open if and only if $U=\varepsilon^{-1}(V)$ for an open set $V$ in $\mathbf{R}^{r}$. The integer $L$ will be regarded as fixed throughout the paper unless it is indicated otherwise.

1.6. Remark. This topology on $E^{r, s}$ is not Hausdorff, but its failure to be Hausdorff may be regarded as compensation for the fact that $\Lambda \mathbf{R}^{L}$ fails to be a field. If $a$ and $b$ are elements in $\Lambda \mathbf{R}^{L}=E^{1,1}$, then $a$ and $b$ have disjoint neighbourhoods if and only if $a-b$ is invertible in $\Lambda \mathbf{R}^{L}$.

1.7. Definitions AND NOtATION. If $R$ and $S$ are sets, let $F(R, S)$ denote the set of functions from $R$ to $S$. If $U$ is an open set in $E^{r, s}$, multiplication in $\Lambda \mathbf{R}^{L}$ gives $F\left(U, \Lambda \mathbf{R}^{L}\right)$ the structure of a graded-commutative algebra. In $\$ 2$ we will define a subalgebra $M^{\infty}\left(U, \Lambda \mathbf{R}^{L}\right)$ of $F\left(U, \Lambda \mathbf{R}^{L}\right)$, called the algebra of smooth functions on $U$. Assuming this definition, define the set of smooth maps from $U$ to another super-Euclidean space $E^{r^{\prime}, s^{\prime}}, M^{\infty}\left(U, E^{r^{\prime}, s^{\prime}}\right)$ to be the subset of $F\left(U, E^{r^{\prime}, s^{\prime}}\right)$ consisting of elements $\mathcal{T}$ such that the function $T$ followed by projection onto any factor of $E^{r^{\prime}, s^{\prime}}$ is in $M^{\infty}\left(U, \Lambda \mathbf{R}^{L}\right)$. If $W$ in $E^{r^{\prime}, s^{\prime}}$ is the image of $U$ under $T, T$ is called a superdiffeomorphism from $U$ to $W$ if $T$ is smooth and has a smooth inverse.

Let $S$ be a topological space. A super-Euclidean chart on $\delta$ is a pair $(U, \phi)$ where $U$ is an open set in $S$ and $\phi$ is a homeomorphism of $U$ with an open set in some super-Euclidean space. A smooth atlas of super-Euclidean charts is a collection $\left\{\left(U_{\alpha}, \phi_{\alpha}\right)\right\}=\mathbb{Q}$ of charts such that $\left\{U_{\alpha}\right\}$ is an open cover of $S$ and

$$
\phi_{\alpha} \phi_{\beta}^{-1}: \phi_{\beta}\left(U_{\alpha} \cap U_{\beta}\right) \rightarrow \phi_{\alpha}\left(U_{\alpha} \cap U_{\beta}\right)
$$

is a superdiffeomorphism for all pairs $\alpha, \beta$. Such an atlas is maximal if, given any chart $(U, \phi)$ such that

$$
\phi \phi_{\beta}^{-1}: \phi_{\beta}\left(U \cap U_{\beta}\right) \rightarrow \phi\left(U \cap U_{\beta}\right)
$$

is a superdiffeomorphism for all $\beta$, then $(U, \phi)$ is in $\mathcal{Q}$.

Finally, a supermanifold $S$ is a topological space together with a maximal smooth atlas of super-Euclidean charts. Let $S$ and $S^{\prime}$ be supermanifolds with atlases $Q$ and $Q^{\prime}$ respectively. A map $T: S \rightarrow S^{\prime}$ is said to be smooth if for every chart $(U, \phi)$ in $\mathbb{Q}$ and every chart $\left(U^{\prime}, \phi^{\prime}\right)$ in $\mathbb{Q}^{\prime}$, the map

$$
\phi^{-1}\left(U \cap T^{-1}\left(U^{\prime}\right)\right) \stackrel{\phi}{\rightarrow} U \cap T^{-1}\left(U^{\prime}\right) \stackrel{T}{\rightarrow} T(U) \cap U^{\prime} \stackrel{\phi^{\prime}}{\rightarrow} \phi^{\prime}\left(T(U) \cap U^{\prime}\right)
$$


is smooth. The category of supermanifolds and smooth maps will be denoted $\delta \Re_{L}$.

Each supermanifold $S$ is associated with an ordinary manifold. The following notation is useful. If $A$ and $B$ are algebras, let $\operatorname{Alg}(A, B)$ denote the space of algebra homomorphisms. If $A$ and $B$ are graded algebras, let $Z_{2} \operatorname{Alg}(A, B)$ denote the space of algebra homomorphisms which respect the grading. The following proposition will be proved in $\$ 3$. fold.

1.8. Proposition. (i) $\operatorname{Alg}\left(M^{\infty}\left(S, \Lambda \mathbf{R}^{L}\right), \mathbf{R}\right)$ can be given the structure of a mani-

(ii) There is a continuous open map

$$
\varepsilon_{S}: S \rightarrow \operatorname{Alg}\left(M^{\infty}\left(S, \Lambda \mathbf{R}^{L}\right), \mathbf{R}\right) .
$$

That part (ii) makes sense depends on Proposition 1.10.

Definition. If $S$ is a supermanifold define the underlying manifold $\bar{S}$ of $S$ to be the manifold $\operatorname{Alg}\left(M^{\infty}\left(\delta, \Lambda \mathbf{R}^{L}\right), \mathbf{R}\right)$.

We can now state the main theorem.

1.9. THEOREM. There exist functors

$$
\begin{aligned}
& H_{L}: \mathcal{G} \mathfrak{K}_{L} \rightarrow \mathcal{S} \mathfrak{K}_{L}, \quad H_{L}(X, A)=\mathbf{Z}_{2} \operatorname{Alg}\left(A(X), \Lambda \mathbf{R}^{L}\right), \\
& M_{L}: \delta \Re_{L} \rightarrow \mathcal{G} \Re_{L}, \quad M_{L}(S)=\left(\bar{S}, M^{\infty}\left(\varepsilon_{S}^{-1}(), \Lambda \mathbf{R}^{L}\right)\right) \text {, }
\end{aligned}
$$

and functorial homomorphisms

$$
\eta: 1_{\mathcal{S} \mathbb{R}_{L}} \rightarrow M_{L} H_{L}, \quad \zeta: 1_{\delta \pi_{L}} \rightarrow H_{L} M_{L},
$$

which establishes the equivalence of the categories $\mathcal{G} \mathfrak{R}_{L}$ and $\mathfrak{S} \mathfrak{R}_{L}$.

So far $L$ has been a fixed integer. If $L^{\prime}$ is another positive integer, we can define categories $\mathcal{S} \mathfrak{K}_{L^{\prime}}$ and $\mathcal{G} \mathfrak{T}_{L^{\prime}}$ in a similar fashion. The resulting categories are related in the following proposition

1.10. Proposition. Let $L$ and $L^{\prime}$ be integers with $L<L^{\prime}$.

(i) $\delta \Re_{L}$ includes as a full subcategory of $\delta \Re_{L^{\prime}}$.

(ii) $\mathcal{G} \mathfrak{M}_{L}$ includes as a full subcategory of $\mathcal{G} \mathfrak{M}_{L^{\prime}}$.

Notice that $\mathcal{S} \mathfrak{K}_{0}$ and $\mathcal{S} \mathfrak{T}_{0}$ are both just the category of smooth manifolds and smooth maps.

Definition-Corollary. Define the category $\mathfrak{S} \mathfrak{\Re}$ to be the direct limit of the categories $\mathfrak{S} \mathfrak{K}_{L}$. Define the category $\mathcal{G} \mathfrak{N}$ to be the direct limit of the categories $\mathcal{G} \mathfrak{N}_{L}$. Then $\mathcal{S} \mathfrak{N}$ and $\mathcal{G} \mathfrak{N}$ are equivalent categories.

Proofs will be given in $\$ 3$.

2. Smooth functions on super-Euclidean space. The method of obtaining smooth functions is based on a technique described by Kostant [8, §2.18]. The guiding principle is that polynomials should approximate smooth functions. 
2.1. Definitions. In $F\left(E^{r, s}, \Lambda \mathbf{R}^{L}\right)$, there are projections $p_{i}, i=1, \ldots, r, \pi_{j}$, $j=1, \ldots, s$ given by

$$
p_{i}\left(u_{1}, \ldots, u_{r}, v_{1}, \ldots, v_{s}\right)=u_{i}, \quad \pi_{j}\left(u_{1}, \ldots, u_{r}, v_{1}, \ldots, v_{s}\right)=v_{j} .
$$

Define the algebra of superpolynomials $P$ to be the (real) subalgebra of $F\left(E^{r, s}, \Lambda \mathbf{R}^{L}\right)$ generated by the projections. Then $P$ is a graded commutative algebra. Let $\operatorname{Sym}\left(p_{i}\right)$ denote the symmetric algebra generated by the elements $p_{i}$, and let $\Lambda\left(\pi_{j}\right)$ denote the exterior algebra generated by the elements $\pi_{j}$. Then $P \cong \operatorname{Sym}\left(p_{i}\right) \otimes \Lambda\left(\pi_{j}\right)$.

Define the space of super-derivations $D$ to be the linear subspace of $\operatorname{End}(P)$ determined by $D=D_{0} \oplus D_{1}$ where

$$
D_{i}=\left\{d \in(\operatorname{End}(P))_{i}: d(a b)=(d a)+(-1)^{i|a|} a d b \text { for all } a, b \text { in } P\right\} .
$$

Define the algebra of superdifferential operators $\mathscr{D}$ to be the subalgebra of $\operatorname{End}(P)$ generated by $D$.

2.2. Notation. Denote by $M(r)$ (or simply $M$ when there is little danger of confusion) the set of $r$-tuples $\left(i_{1}, \ldots, i_{r}\right)$ of nonnegative integers. Denote by $N(s)$ (or simply $N$ ) the set of $s$-tuples $\left(j_{1}, \ldots, j_{s}\right)$ where each $j_{k}$ is either 0 or 1 . If $\mu=\left(i_{1}, \ldots, i_{r}\right)$ in $M$ and $\nu=\left(j_{1}, \ldots, j_{s}\right)$ in $N$ then write

$$
p \pi(\mu, \nu)=p_{1}^{i_{1}} \cdots p_{r}^{i_{r}} \pi_{1}^{j_{i}} \cdots \pi_{s}^{j_{s}}
$$

Let $\partial p_{i}$ be the element of $D$ determined by $\partial p_{i} p_{j}=\delta_{i j}$ and $\partial p_{i} \pi_{j}=0$ for all $j=1, \ldots, s$. Similarly let $\partial \pi_{j}$ be the superderivation corresponding to $\pi_{j}$. Write

$$
d(\mu, \nu)=\partial p_{1}^{i_{1}} \cdots \partial p_{r}^{i_{r}} \partial \pi_{s}^{j_{s}} \ldots \partial \pi_{1}^{j_{1}}
$$

We can define a partial ordering on $M \times N$ be setting $(\mu, \nu)<\left(\mu^{\prime}, \nu^{\prime}\right)$ if $i_{h}<i_{h}^{\prime}$ and $j_{k}<j_{k}^{\prime}$ for every $h=1, \ldots, r$ and $k=1, \ldots, s$. We can also define the length of elements of $M, N$ and $M \times N$, denoted by || via

$$
|\mu|=\sum_{h=1}^{r} i_{h}, \quad|\nu|=\sum_{k=1}^{s} j_{k} \text { and }|(\mu, \nu)|=|\mu|+|\nu| .
$$

2.3. Remarks. $D$ is a free $P$ module generated by the elements $\partial p_{i}, \partial \pi_{j}$. $\mathscr{D}$ is a free $P$-module generated by the elements $d(\mu, \nu)$ for $(\mu, \nu)$ in $M(r) \times N(s)$.

Definitions. Define Diff to be the subalgebra of $\mathscr{D}$ generated (over the real numbers) by the elements $d(\mu, \nu)$, for $(\mu, \nu)$ in $M(r) \times N(s)$.

Let $P^{*}$ denote the subspace of the full linear dual $P^{\prime}$ of $P$ given by

$$
P^{*}=\left\{a \in P^{\prime}: \operatorname{ker} a \text { contains an ideal of finite codimension }\right\} \text {. }
$$

If $a$ is in $P^{*}$ and $p$ is in $P$, denote the evaluation of $a$ on $p$ by $\langle a, p\rangle$. The properties of this space that will be used are summarized in the following proposition.

2.4. Proposition. (i) There is a right action of $\mathscr{D}$ on $P^{*}$ given by $\langle a . d, p\rangle=$ $\langle a, d p\rangle$ for $a$ in $P^{*}, d$ in $\mathscr{D}$ and $p$ in $P$.

(ii) There is an inclusion $\mathbf{R}^{r} \subset P^{*}$ given by

$$
\left\langle\left(x_{1}, \ldots, x_{r}\right), p\right\rangle=p\left(x_{1}, \ldots, x_{r}, 0, \ldots, 0\right)
$$

regarding $\left(x_{1}, \ldots, x_{r}, 0, \ldots, 0\right)$ as an element of $E^{r, s}$. This inclusion induces a map

$$
\phi: P \rightarrow C^{\infty}(\mathbf{R}) \text {. }
$$

(iii) $P^{*}$ is a free Diff module generated by elements of $\mathbf{R}^{r}$. 
Proof. Parts (i) and (ii) are not hard. For part (iii), notice that $P$ can be given the structure of a Hopf algebra with antipode where the comultiplication $\Delta$ is determined by

$$
\Delta\left(p_{i}\right)=1 \otimes p_{i}+p_{i} \otimes 1, \quad \Delta\left(\pi_{j}\right)=1 \otimes \pi_{j}+\pi_{j} \otimes 1,
$$

and the antipode $s$ is determined by

$$
s\left(p_{i}\right)=-p_{i}, \quad s\left(\pi_{j}\right)=-\pi_{j} .
$$

Then $P^{*}$ is also a Hopf algebra with antipode. Let $G$ denote the grouplike elements of $P^{*}$ and let $U(H)$ denote the universal enveloping algebra of the Lie algebra $H$ of primitive elements of $P^{*}$. Then there is an isomorphism of vector spaces

$$
\alpha: \mathbf{R} G \otimes U(H) \rightarrow P^{*} .
$$

(See Sweedler [10] for basic properties of Hopf algebras.) Notice that $G=\mathbf{R}^{r}$ and $U(H)=$ Diff. The result follows by observing that $\alpha$ is the map determined by the action of Diff on $P^{*}$.

2.5. Definition-Proposition. For an open set $U \subset E^{r, s}$, define

$$
P^{*}(U)=\varepsilon(U) \cdot \text { Diff }
$$

and let $P^{*}(U)^{\prime}$ denote the full linear dual of $P^{*}(U)$. Then:

(i) $P^{*}(U)^{\prime}$ is an algebra containing $P$.

(ii) Diff acts on $P^{*}(U)^{\prime}$ on the left.

(iii) The homomorphism $\phi: P \rightarrow C^{\infty}\left(\mathbf{R}^{r}\right)$ extends to a homomorphism

$$
\tilde{\phi}: P^{*}(U)^{\prime} \rightarrow F(\varepsilon(U), \mathbf{R}) \text {. }
$$

(iv) There is an algebra homomorphism

$$
I: P^{*}(U)^{\prime} \rightarrow F\left(U, \Lambda \mathbf{R}^{L}\right)
$$

given by

$$
I(f)(u, v)=\sum_{|\mu, \nu|<L} c(\mu) \tilde{\phi}(d(\mu, \nu) f) \varepsilon(u) p \pi(\mu, \nu)(u-\varepsilon(u), r),
$$

where

$$
c(\mu)=\frac{1}{i_{1} ! \cdots i_{r} !} \text { for } \mu=\left(i_{1}, \ldots, i_{r}\right) .
$$

Moreover, for an element $q$ in $P<P^{*}(U)^{\prime}, I(q)(u, v)=q(u, v)$.

Proof. Parts (i), (ii) and (iii) are easy. To check that $I$ of part (iv) is an algebra homomorphism, let $f$ and $g$ be elements of $P^{*}(U)^{\prime}$ and compute $I(f g)$ directly, making use of the following identities.

$$
\begin{aligned}
d(\mu, \nu) f g= & \sum_{\left(\mu^{\prime}, \nu^{\prime}\right)+\left(\mu^{\prime \prime}, \nu^{\prime \prime}\right)=(\mu, \nu)}(-1)^{\bar{\gamma}\left(\nu^{\prime}, \nu^{\prime \prime}\right)+\left|\nu^{\prime \prime}\right|} \mid{ }^{\prime} d\left(\mu^{\prime}, \nu^{\prime}\right) f d\left(\mu^{\prime \prime}, \nu^{\prime \prime}\right) g, \\
& p \pi(\mu, \nu)=(-1)^{\gamma\left(\nu^{\prime}, \nu^{\prime \prime}\right)} p \pi\left(\mu^{\prime}, \nu^{\prime}\right) p \pi\left(\mu^{\prime \prime}, \nu^{\prime \prime}\right),
\end{aligned}
$$

where $\gamma\left(\nu^{\prime}, \nu^{\prime \prime}\right)$ and $\bar{\gamma}\left(\nu^{\prime}, \nu^{\prime \prime}\right)$ are determined by

$$
\begin{aligned}
p \pi(0, \nu) & =(-1)^{\gamma\left(\nu^{\prime}, \nu^{*}\right)} p \pi\left(0, \nu^{\prime}\right) p \pi\left(0, \nu^{\prime \prime}\right), \\
d(0, \nu) & =(-1)^{\bar{\gamma}\left(\nu^{\prime}, \nu^{\prime \prime}\right)} d\left(0, \nu^{\prime}\right) d\left(0, \nu^{\prime \prime}\right) .
\end{aligned}
$$


A direct computation shows that

$$
(-1)^{\gamma\left(\nu^{\prime}, \nu^{\prime \prime}\right)+\bar{\gamma}\left(\nu^{\prime}, \nu^{\prime \prime}\right)+\left|\nu^{\prime \prime}\right||\emptyset|}=1 \text { when }\left|\nu^{\prime}\right|=|f| \text {. }
$$

2.6. Definition. Let $U$ be an open set in $E^{r, s}$. Define the set of smooth functions from $U$ to $\Lambda \mathbf{R}^{L}, M^{\infty}\left(U, \Lambda \mathbf{R}^{L}\right)$ by

$$
M^{\infty}\left(U, \Lambda \mathbf{R}^{L}\right)=\left\{f \in P^{*}(U)^{\prime}: \tilde{\phi}(d f) \text { is in } C^{\infty}(\varepsilon(U)) \text { for all } d \text { in } \mathscr{D}\right\} .
$$

The properties of $M^{\infty}\left(U, \Lambda \mathbf{R}^{L}\right)$ are summarized in the following proposition.

2.7. Proposition. (i) $M^{\infty}\left(U, \Lambda \mathbf{R}^{L}\right)$ is closed under multiplication.

(ii) $M^{\infty}\left(U, \Lambda \mathbf{R}^{L}\right)=\left\{f \in P^{*}(U)^{\prime}: I(d f)\right.$ is continuous for every $d$ in $\left.\mathscr{Q}\right\}$.

(iii) If $\partial(\mu)$ denotes the ordinary differential operator

$$
\frac{\partial^{i_{1}}}{\partial x_{1}} \cdots \frac{\partial^{i_{r}}}{\partial x_{r}}
$$

on $C^{\infty}(\varepsilon(U))$, where $\mu=\left(i_{1}, \ldots, i_{r}\right)$, then for $f$ in $M^{\infty}\left(U, \Lambda \mathbf{R}^{L}\right)$,

$$
\tilde{\phi}(d(\mu, \nu) f)=\partial \mu \tilde{\phi}(d(0, \nu) f) \text {. }
$$

(iv) The homomorphism I of 2.5(iv) is injective on $M^{\infty}\left(U, \Lambda \mathbf{R}^{L}\right)$.

(v) The map $\psi: M^{\infty}\left(U, \Lambda \mathbf{R}^{L}\right) \rightarrow C^{\infty}(\varepsilon(U)) \otimes \Lambda\left(\pi_{j}\right)$ given by

$$
\psi(f)=\sum_{(0, \nu)} \tilde{\phi}(d(0, \nu) f) \otimes p \pi(0, \nu)
$$

is an isomorphism of algebras.

INDICATIONS OF PROOFS. (i) This follows since $\mathscr{D}$ is generated by superderivations, since $\tilde{\phi}$ is an algebra homomorphism, and since $C^{\infty}(\varepsilon(U))$ is closed under multiplication.

(iii) For an open set $V$ in $\mathbf{R}^{r}$, define the space $C^{\infty}(V)^{*}$ to be the subspace of the full linear dual $C^{\infty}(V)^{\prime}$ of $C^{\infty}(V)$ given by

$C^{\infty}(V)^{*}=\left\{\alpha \in C^{\infty}(V)^{\prime}:\right.$ where $\alpha$ contains an ideal of finite codimension $\}$.

Since $\phi: P \rightarrow C^{\infty}(V)$ separates points in $V$, the induced map

is injective. In fact, if $V=\varepsilon(U)$, then

$$
\phi^{*}: C^{\infty}(V)^{*} \rightarrow P^{*}
$$

$$
\phi^{*}: C^{\infty}(V)^{*} \rightarrow P^{*}(U)
$$

If $x$ is in $V$, denote by $x . \partial(\mu)$ the element of $C^{\infty}(V)^{*}$ given by $\langle x . \partial(\mu), h\rangle=$ $\partial(\mu) h(x)$ for any element $h$ in $C^{\infty}(V)$. It is not hard to see that

$$
\phi^{*}(x . \partial(\mu))=x . d(\mu, 0) \text {. }
$$

Taking the full linear dual of $C^{\infty}(V)^{*}$ and $P^{*}(U)$, we have a map of algebras

$$
\phi^{* \prime}: P^{*}(U)^{\prime} \rightarrow C^{\infty}(V)^{* \prime} \text {. }
$$

The map $\tilde{\phi}: M^{\infty}\left(U, \Lambda \mathbf{R}^{L}\right) \rightarrow C^{\infty}(V)$ is just the restriction of $\phi^{* \prime}$ to the inverse image of $C^{\infty}(V) \subset C^{\infty}(V)^{* \prime}$ under $\phi^{* \prime}$. The result then follows from (A).

(iv) Suppose that $I(f)=0$ for some $f$ in $M^{\infty}\left(U, \Lambda \mathbf{R}^{L}\right)$. Then $\tilde{\phi}(d(\mu, \nu) f)$ is identically zero whenever $|\mu, \nu|<L$. If $f$ is not zero, then for some $(\mu, \nu)$ and some $x$ in $\varepsilon(U),\langle x . d(\mu, \nu), f\rangle=\tilde{\phi}(d(\mu, \nu) f(x))$ is nonzero. Choose $\left(\mu_{0}, \nu_{0}\right)$ such that 
$\tilde{\phi}\left(d\left(\mu_{0}, \nu_{0}\right) f\right)$ is not identically zero on $\varepsilon(U)$, and such that if $\tilde{\phi}(d(\mu, \nu) f)$ is not identically zero; then $|\mu, \nu|>\left|\mu_{0} \nu_{0}\right|$. Notice that $\left|\mu_{0}\right|>0$, since $\left|\mu_{0}, \nu_{0}\right|>L>s$. Then $\tilde{\phi}\left(d\left(\mu_{0}, \nu_{0}\right) f\right)=\partial\left(\mu_{0}\right) \tilde{\phi}\left(d\left(0, \nu_{0}\right) f\right)$ by part (iii) which implies that $\tilde{\phi}\left(d\left(0, \nu_{0}\right) f\right)$ is not identically zero. But $\left|0, \nu_{0}\right|<\left|\mu_{0}, \nu_{0}\right|$ which contradicts the minimality of $\left(\mu_{0}, \nu_{0}\right)$.

(v) The proof is by direct computation.

(ii) Using part (iii), notice that

$$
M^{\infty}\left(U, \Lambda \mathbf{R}^{L}\right)=\left\{f \in P^{*}(U)^{\prime}: \tilde{\phi}(d f) \text { is continuous for every } d \text { in } \mathscr{D}\right\} .
$$

The result then is a consequence of the following characterization of continuous maps in $F\left(U, \Lambda \mathbf{R}^{L}\right)$.

2.8. LemMa. Let $U, U^{\prime}$ be open sets in $E^{r, s}$ and $E^{r^{\prime}, s^{\prime}}$ respectively. Then an element $f$ in $F\left(U, U^{\prime}\right)$ is continuous if and only if there exists a continuous map $h: \varepsilon(U) \rightarrow$ $\varepsilon\left(U^{\prime}\right)$ such that $\varepsilon \circ f=h \circ \varepsilon$.

Proof. Assume $f$ is continuous. Notice that if $u_{1}$ and $u_{2}$ are in $U$ and $\varepsilon\left(u_{1}\right)=$ $\varepsilon\left(u_{2}\right)$, then $\varepsilon f\left(u_{1}\right)=\varepsilon f\left(u_{2}\right)$. If not, there would be open sets $O_{1}$ and $O_{2}$ in $U^{\prime}$ with $f\left(u_{1}\right) \in O_{1}$ and $f\left(u_{2}\right) \in O_{2}$ and $O_{1} \cap O_{2}=\varnothing$. But then $u_{1} \in f^{-1}\left(O_{1}\right)$ and $u_{2} \in$ $f^{-1}\left(O_{2}\right)$. Since $\varepsilon\left(u_{1}\right)=\varepsilon\left(u_{2}\right)$ and $u_{1}$ and $u_{2}$ cannot be separated by open sets so $f^{-1}\left(O_{1}\right)$ and $f^{-1}\left(O_{2}\right)$ have nonempty intersection, which contradicts the fact that $O_{1}$ and $O_{2}$ are disjoint. Then $h: \varepsilon(U) \rightarrow \varepsilon\left(U^{\prime}\right)$ given by $h(x)=\varepsilon f(u)$ for $u$ in $\varepsilon^{-1}(x)$ is a well-defined map. It is not hard to show that $h$ is continuous and that $\varepsilon \circ f=h \circ \varepsilon$. The implication the other way is also not hard to see.

2.9. RemARK. $M^{\infty}\left(U, \Lambda \mathbf{R}^{L}\right)$ and $C^{\infty}(\varepsilon(U)) \otimes \Lambda\left(\pi_{j}\right)$ can be topologized so that $\psi$ is a homeomorphism. Let $\left\{K_{i}\right\}$ be a collection of compact sets indexed by the natural numbers such that $\cup_{i} K_{i}=U$ and $K_{i} \subset$ Int $K_{i+1}$. Define a family of seminorms on $C^{\infty}(\varepsilon(U))$,

$$
\rho_{t}(f)=\max \left\{|\partial(\mu) f(x)|:|\mu| \leqslant t, x \in K_{t}\right\} .
$$

A local base for a topology on $C^{\infty}(\varepsilon(U))$ is given by

$$
V_{t}=\left\{f \in C^{\infty}(\varepsilon(U)): \rho_{t}(f)<1 / t\right\} .
$$

Finally, give $C^{\infty}(\varepsilon(U)) \otimes \Lambda\left(\pi_{j}\right)$ the topology of a product of $2^{s}$ copies of $C^{\infty}(\varepsilon(U))$.

On $M^{\infty}\left(U, \Lambda \mathbf{R}^{L}\right)$, define seminorms

$$
\sigma_{t}(g)=\max \left\{|\tilde{\phi}(d(\mu, \nu) g)(x)|: x \in K_{t},|\mu, \nu|<t\right\} .
$$

Give $M^{\infty}\left(U, \Lambda \mathbf{R}^{L}\right)$ the topology determined by the local base corresponding to the seminorms $\left\{\sigma_{t}\right\}$. With respect to these topologies we have the following proposition.

2.10. Proposition. (i) The map $\psi$ of 2.7(v) is a homeomorphism.

(ii) $M^{\infty}\left(U, \Lambda \mathbf{R}^{L}\right)$ is complete and $P$ is dense in $M^{\infty}\left(U, \Lambda \mathbf{R}^{L}\right)$.

Proof. Part (i) is straightforward. Part (ii) is a consequence of the corresponding nontrivial results for $C^{\infty}(\varepsilon(U))$. See $[9$, p. 31] and [11, p. 189]. 
Let $U_{i}$ be an open set in $E^{r_{i} s_{i}}, i=1,2,3$. Using the definition at $M^{\infty}\left(U, U^{\prime}\right)$ given in 1.7 , the following proposition guarantees that the composition of two smooth maps is again smooth.

Proposition. Let $f$ be in $M^{\infty}\left(U_{1}, U_{2}\right)$ and let $g$ be in $M^{\infty}\left(U_{2}, U_{3}\right)$. Then $g \circ f$ is in $M^{\infty}\left(U_{1}, U_{3}\right)$.

Proof. Since $g \circ f$ will be in $M^{\infty}\left(U_{1}, U_{3}\right)$ if and only if $g \circ f$, composed with any projection, is in $M^{\infty}\left(U_{1}, \Lambda \mathbf{R}^{L}\right)$. It is sufficient to consider the case where $g$ is in $M^{\infty}\left(U_{2}, \Lambda \mathbf{R}^{L}\right)$. If $g$ is a projection, $g \circ f$ is in $M^{\infty}\left(U_{1}, \Lambda \mathbf{R}^{L}\right)$ since $f$ is smooth. If $g$ is a superpolynomial, then $g \circ f$ is the sum of products of element in $M^{\infty}\left(U_{1}, \Lambda \mathbf{R}^{L}\right)$ and hence $g \circ f$ is in $M^{\infty}\left(U_{1}, \Lambda \mathbf{R}^{L}\right) . M^{\infty}\left(U_{1}, U_{2}\right)$ has a topology induced by the inclusion $M^{\infty}\left(U_{1}, U_{2}\right) \rightarrow\left(M^{\infty}\left(U_{1}, \Lambda \mathbf{R}^{L}\right)\right)^{r_{2}+s_{2}}$ given by the projections. The algebra $P\left(r_{2}, s_{2}\right)$ of superpolynomials on $E^{r_{2}, s_{2}}$ has a topology as subset of $M^{\infty}\left(U_{2}, \Lambda \mathbf{R}^{L}\right)$. The map determined by composition

$$
C: M^{\infty}\left(U_{1}, U_{2}\right) \times P\left(r_{2}, s_{2}\right) \rightarrow M^{\infty}\left(U, \Lambda \mathbf{R}^{L}\right)
$$

is continuous. Now if $g$ is an arbitrary element of $M^{\infty}\left(U_{2}, \Lambda \mathbf{R}^{L}\right)$, let $\left\{g_{i}\right\}$ be a sequence of superpolynomials in $P\left(r_{2}, s_{2}\right)$ such that $g_{i} \rightarrow g$. Then $g_{i} \circ f$ is a Cauchy sequence in $M^{\infty}\left(U, \Lambda \mathbf{R}^{L}\right)$ converging to $g \circ f$.

Supermanifolds finally can be defined as in 1.7. We have the following corollary of Proposition 2.10.

2.11. COROLlARY. Supermanifolds and smooth maps between supermanifolds form a category, denoted by $\delta \Re_{L}$.

2.12. REMARK. The definition of smooth functions on super-Euclidean space is chosen to make the main theorem true. Another reasonable description of smooth maps following this same procedure would be to define an algebra $S P$ to be the algebra of superpolynomials with coefficients in $\Lambda \mathbf{R}^{L}$, that is, the algebras over $\Lambda \mathbf{R}^{L}$ generated by the projections $p_{i}$ and $\pi_{j}$. Then $S P^{*}, S P^{*}(U)^{\prime}$ and $S M^{\infty}\left(U, \Lambda \mathbf{R}^{L}\right)$ can be defined by analogy with $P^{*}, P(U)^{\prime}$ and $M^{\infty}\left(U, \Lambda \mathbf{R}^{L}\right)$. It turns out that

$$
\begin{aligned}
S P & \cong \Lambda \mathbf{R}^{L} \otimes P \\
S M^{\infty}\left(U, \Lambda \mathbf{R}^{L}\right) & \cong \Lambda \mathbf{R}^{L} \otimes M^{\infty}\left(U, \Lambda \mathbf{R}^{L}\right) .
\end{aligned}
$$

The advantage of $S M^{\infty}\left(U, \Lambda \mathbf{R}^{L}\right)$ over $M^{\infty}\left(U, \Lambda \mathbf{R}^{L}\right)$ is that $S M^{\infty}\left(U, \Lambda \mathbf{R}^{L}\right)$ contains the constant maps: $U \rightarrow u_{0} \in \Lambda \mathbf{R}^{L}$. This definition also seems to coincide exactly with de Witt's definition of smooth maps. In practice, $S M^{\infty}\left(U, \Lambda \mathbf{R}^{L}\right)$ may be the more useful definition.

\section{Proof of the main theorem.}

3.1. Proof of Theorem 1.9. Step 1. Definition of $H_{L}: \mathcal{G} \mathfrak{R}_{L} \rightarrow \mathcal{S} \mathfrak{K}_{L}$ on objects.

Let $(X, A)$ be a graded manifold. It must be shown that

$$
H(X, A)=\mathbf{Z}_{2} \operatorname{Alg}\left(A X, \Lambda \mathbf{R}^{L}\right)
$$


is a supermanifold. $H(X, A)$ is a topological space where open sets in $H(X, A)$ are the images of the maps

$$
\mathbf{Z}_{2} \operatorname{Alg}\left(A(V), \Lambda \mathbf{R}^{L}\right) \rightarrow \mathbf{Z}_{2} \operatorname{Alg}\left(A X, \Lambda \mathbf{R}^{L}\right)
$$

induced by the restriction maps $A(X) \rightarrow A(V)$ for an open set $V$ in $X$. We can choose a cover $\left\{V_{i}\right\}$ of $X$ such that

$$
T_{i}: A\left(V_{i}\right) \cong C^{\infty}\left(V_{i}\right) \otimes \Lambda \mathbf{R}^{s} .
$$

We can assume that each $V_{i}$ is homeomorphic to an open set in $\mathbf{R}^{r}$. The following lemma is then sufficient to ensure that $H(X, A)$ is covered by open sets homeomorphic to open sets in $E^{r, s}$.

\subsection{Lemma. Let $V$ be an open set in $\mathbf{R}^{r}$. Then there is a bijection}

$$
Q: \mathbf{Z}_{2} \operatorname{Alg}\left(C^{\infty}(V) \otimes \Lambda \mathbf{R}^{s}, \Lambda \mathbf{R}^{L}\right) \rightarrow \varepsilon^{-1}(V) \subset E^{r, s}
$$

given by

$$
Q(\alpha)=\left(\alpha\left(x_{1}\right), \ldots, \alpha\left(x_{r}\right), \alpha\left(\pi_{1}\right), \ldots, \alpha\left(\pi_{j}\right)\right)
$$

where $x_{i}: V \rightarrow \mathbf{R}$ is projection onto the ith coordinate, and $\pi_{j}, j=1, \ldots, s$, is a basis for $\mathbf{R}^{s}$.

Proof. Since $\alpha$ is a homomorphism preserving the $\mathbf{Z}_{2}$-grading, each $\alpha\left(x_{i}\right)$ is in $\left(\Lambda \mathbf{R}^{L}\right)_{0}$ and each $\alpha\left(\pi_{j}\right)$ is in $\left(\Lambda \mathbf{R}^{L}\right)_{1}$ so $Q(\alpha)$ is in $E^{r, s}$. To see that $\varepsilon(Q(\alpha))$ is in $V$, consider the algebra homomorphism $\rho: C^{\infty}(V) \rightarrow \mathbf{R}$ given by $\rho(f)=\varepsilon \alpha(f)$. Since $\rho$ is an algebra homomorphism, $\rho(f)=f\left(x_{\rho}\right)$ for some point $x_{\rho}$ in $V$, and for all $f$ in $C^{\infty}(V)$. But $\rho\left(x_{i}\right)=\varepsilon \alpha\left(x_{i}\right)$ which implies that $\varepsilon Q(\alpha)=x_{\rho}$ which is in $V$.

Injectivity of $Q$. Notice that an algebra homomorphism $\alpha: C^{\infty}(V) \otimes \Lambda \mathbf{R}^{s} \rightarrow$ $\Lambda \mathbf{R}^{L}$ is a continuous map, since $\alpha$ is continuous if $\varepsilon \circ \alpha$ is continuous, and $\varepsilon \circ \alpha=\rho=$ evaluation at $x_{\rho}$, which is a continuous map. Suppose now that $Q(\alpha)=Q\left(\alpha^{\prime}\right)$. Then $\alpha\left(x_{i}\right)=\alpha^{\prime}\left(x_{i}\right)$ and $\alpha^{\prime}\left(\pi_{j}\right)=\alpha\left(\pi_{j}\right)$ for all appropriate $i$ and $j$, and hence $\alpha(q)=\alpha^{\prime}(q)$ for any "polynomial" $q$ in the $x_{i}$ and $\pi_{j}$. Since such "polynomials" are dense in $C^{\infty}(V) \otimes \Lambda \mathbf{R}^{s}$, and since $\alpha$ and $\alpha^{\prime}$ are continuous, $\alpha=\alpha^{\prime}$.

Surjectivity of $Q$. Let $(u, v)=\left(u_{1}, \ldots, u_{r}, v_{1}, \ldots, v_{s}\right)$ be in $\varepsilon^{-1}(V)$. Define a $\operatorname{map} \alpha_{(u, v)}: C^{\infty}(V) \otimes \Lambda \mathbf{R}^{s} \rightarrow \Lambda \mathbf{R}^{L}$ by

$$
\alpha_{(u, v)}(f \otimes p \pi(0, \nu))=\sum_{|\mu|<L} c(\mu) \partial(\mu) f(\varepsilon(u)) p \pi(\mu, \nu)(u-\varepsilon(u), v)
$$

where

$$
c(\mu)=\frac{1}{i_{1} ! \cdots i_{r} !} \quad \text { for } \mu=\left(i_{1}, \ldots, i_{r}\right) .
$$

The proof that this is an algebra homomorphism is similar to the proof of 2.5(iv). Again the calculation is unpleasant and is omitted. It is immediate that $Q\left(\alpha_{(u, v)}\right)=$ $(u, v)$.

In order to show that $H(X, A)$ is a supermanifold, it remains to show that the isomorphisms

$$
T_{i} T_{j}^{-1}: C^{\infty}\left(V_{i} \cap V_{j}\right) \otimes \Lambda \mathbf{R}^{s} \rightarrow C^{\infty}\left(V_{i} \cap V_{j}\right) \otimes \Lambda \mathbf{R}^{s}
$$


give rise to superdiffeomorphisms. This will be a consequence of Lemma 3.4 and the remark following Lemma 3.4.

3.3. Step 2. Definition of $H_{L}$ on morphisms. Let $(X, A)$ and $(Y, B)$ be graded manifolds. If $J:(X, A) \rightarrow(Y, B)$ is a morphism of graded manifolds, then

$$
\sigma: B(Y) \rightarrow A(X)
$$

is a homomorphism of $\mathbf{Z}_{2}$-graded algebras, and thus induces a map (of sets) given by

$$
H_{L}(\sigma): \mathbf{Z}_{2} \operatorname{Alg}\left(A(X), \Lambda \mathbf{R}^{L}\right) \rightarrow \mathbf{Z}_{2} \operatorname{Alg}\left(B(Y), \Lambda \mathbf{R}^{L}\right)=H_{L}(\sigma)(\alpha)=\alpha \circ \sigma .
$$

The problem of deciding whether $H(\sigma)$ is smooth reduces to the following lemma.

\subsection{LEMMA. Let $\sigma$ be a homomorphism of $\mathbf{Z}_{2}$-graded algebras} $\sigma: C^{\infty}(\mathbf{R}) \otimes \Lambda \mathbf{R} \rightarrow C^{\infty}(V) \otimes \Lambda \mathbf{R}^{s}$ for an open set $V$ in $\mathbf{R}^{s}$.

Then the map induced by $\sigma$,

$$
H_{L}(\sigma): \varepsilon^{-1}(V) \rightarrow \Lambda \mathbf{R}^{L}, \quad \varepsilon^{-1}(V) \subset E^{r, s}
$$

is smooth.

Proof. Define an element $g$ in $P^{*}\left(\varepsilon^{-1}(V)\right)^{\prime}$ as follows. Let $x \in C^{\infty}(\mathbf{R})$ denote the function determined by $x(t)=t$. Let $y$ be a generator of $\Lambda \mathbf{R}$. Define $g$ by setting

$$
\langle x . d(\mu, \nu), g\rangle=\partial(\mu) \sigma(x) d(0, \nu) \sigma(y) .
$$

Then it is not hard to see that $g$ is in $M^{\infty}\left(\varepsilon^{-1}(V), \Lambda \mathbf{R}^{L}\right)$. Finally by evaluation $I(g)$ and $H_{L}(\sigma)$ on elements in $\varepsilon^{-1}(V)$, it can be shown that $I(g)=H_{L}(\sigma)$.

REMARK. If $\sigma^{\prime}:(Y, B) \rightarrow(Z, C)$ is another morphism of graded manifolds, it is clear that $H_{L}\left(\sigma^{\prime}\right) H_{L}(\sigma)=H_{L}\left(\sigma^{\prime}, \sigma\right)$ and $H$ is a well-defined functor.

3.5. Step 3. Definition of $M_{L}: \delta \mathfrak{K}_{L} \rightarrow \mathcal{G} \mathfrak{N}_{L}$ on objects. We begin by proving Proposition 1.8, which defines the underlying manifold of a supermanifold $S$.

Let $\left\{\left(U_{\alpha}, \phi_{\alpha}\right)\right\}$ be an atlas for $S$. There are restriction homomorphisms

$$
\rho_{\alpha}: M^{\infty}\left(S, \Lambda \mathbf{R}^{L}\right) \rightarrow M^{\infty}\left(U_{\alpha}, \Lambda \mathbf{R}^{L}\right)
$$

which induce an injection

$$
\begin{aligned}
& \rho: M^{\infty}\left(S, \Lambda \mathbf{R}^{L}\right) \rightarrow\left\{\left(f_{\alpha}\right) \in \prod_{\alpha} M^{\infty}\left(U_{\alpha}, \Lambda \mathbf{R}^{L}\right): f_{\alpha}(x)=f_{\beta}(x)\right. \\
&\text { for all } \left.x \text { in } U_{\alpha} \cap U_{\beta}, \text { for all pairs } \alpha, \beta\right\} .
\end{aligned}
$$

If $a_{\alpha}: M^{\infty}\left(U, \Lambda \mathbf{R}^{L}\right) \rightarrow \mathbf{R}$ is an algebra homomorphism, $a_{\alpha}$ induces an algebra homomorphism

$$
a_{\alpha} \rho_{\alpha}: M^{\infty}\left(S, \Lambda \mathbf{R}^{L}\right) \rightarrow M^{\infty}\left(U_{\alpha}, \Lambda \mathbf{R}^{L}\right) \rightarrow \mathbf{R} .
$$

We then have a map

$$
\bigcup_{\alpha} \operatorname{Alg}\left(M^{\infty}\left(U_{\alpha}, \Lambda \mathbf{R}^{L}\right), \mathbf{R}\right) \rightarrow \operatorname{Alg}\left(M^{\infty}\left(S, \Lambda \mathbf{R}^{L}\right), \mathbf{R}\right)
$$

which induces a bijection

$$
\bigcup_{\alpha} \operatorname{Alg}\left(M^{\infty}\left(U_{\alpha}, \Lambda \mathbf{R}^{L}\right), \mathbf{R}\right) / \sim \operatorname{Alg}\left(M^{\infty}\left(S, \Lambda \mathbf{R}^{L}\right), \mathbf{R}\right)
$$


where, if $a_{\alpha}, b_{\beta}$ are in $\operatorname{Alg}\left(M^{\infty}\left(U_{\alpha}, \Lambda \mathbf{R}^{L}\right), \mathbf{R}\right)$ and $\operatorname{Alg}\left(M^{\infty}\left(U_{\beta}, \Lambda \mathbf{R}^{L}\right), \mathbf{R}\right)$ respectively, $a_{\alpha} \sim b_{\beta}$ if there exists $c_{\alpha \beta}$ in $\operatorname{Alg}\left(M^{\infty}\left(U_{\alpha} \cap U_{\beta}, \Lambda \mathbf{R}^{L}\right), \mathbf{R}\right)$ such that $a_{\alpha}=$ $c_{\alpha \beta} \rho_{\beta}$ and $b_{\beta}=c_{\alpha \beta} \rho_{\alpha}$. Notice that $\operatorname{Alg}\left(M^{\infty}\left(U_{\alpha}, \Lambda \mathbf{R}^{L}\right), \mathbf{R}\right)$ can be identified with $\varepsilon \circ \phi\left(U_{\alpha}\right)$. Then

$$
\operatorname{Alg}\left(M^{\infty}\left(S, \Lambda \mathbf{R}^{L}\right), \mathbf{R}\right)=\bar{S} \cong \bigcup_{\alpha} \varepsilon \circ \phi_{\alpha}\left(U_{\alpha}\right) / \sim^{\prime},
$$

where $a_{\alpha} \sim{ }^{\prime} b_{\beta}$ if $a_{\alpha}=h_{\alpha \beta} b_{\beta}$, where

$$
h_{\alpha \beta}: \varepsilon\left(\phi_{\alpha}\left(U_{\alpha} \cap U_{\beta}\right)\right) \rightarrow \varepsilon\left(\phi_{\beta}\left(U_{\alpha} \cap U_{\beta}\right)\right)
$$

Using 2.7(ii), it is possible to show that $h_{\alpha \beta}$ is a diffeomorphism. This gives $\bar{S}$ a smooth structure.

Define $\varepsilon_{s}$ by setting

$$
\varepsilon_{S}(u)(f)=\varepsilon \circ f(u) \text { for any element } f \text { in } M^{\infty}\left(S, \Lambda \mathbf{R}^{L}\right) .
$$

It is not hard to check that $\varepsilon_{S}$ has the desired properties.

In order to check that $M_{L}(S)=\left(\bar{S}, M^{\infty}\left(\varepsilon_{S}^{-1}, \Lambda \mathbf{R}^{L}\right)\right)$ is a graded manifold, it is sufficient to show that the sheaf $M^{\infty}\left(\varepsilon_{S}^{-1}(), \Lambda \mathbf{R}^{L}\right)$ satisfies the local triviality condition. To see this, choose an atlas $\left\{\left(U_{\alpha}, \phi_{\alpha}\right)\right\}$ at $S$. Then the open cover $\left\{\varepsilon_{S}\left(U_{\alpha}\right)\right\}$ has the desired properties, by 2.7(v) and the following comment 3.6.

3.6. Step 4. Definition of $M_{L}$ on morphisms. Let $R: S \rightarrow S^{\prime}$ be a smooth map between two supermanifolds. It is sufficient to show that

$$
M_{L}(R): M^{\infty}\left(S^{\prime}, \Lambda \mathbf{R}^{L}\right) \rightarrow M^{\infty}\left(S, \Lambda \mathbf{R}^{L}\right)
$$

is a well-defined map of algebras. Well-definition is a consequence of the fact that the composition of smooth maps is a smooth map. The fact that $M_{L}(R)$ preserves the algebraic structure is immediate.

It is also trivial to check that if $R^{\prime}: S^{\prime} \rightarrow S^{\prime \prime}$ is another morphism of supermanifolds, $M_{L}\left(R^{\prime} \circ R\right)=M_{L}(R) \circ M_{L}\left(R^{\prime}\right)$.

Step 5. Equivalence.

3.7. Definition of $\eta$. For a graded manifold $(X, A)$, define $\xi: M_{L} H_{L}(X, A) \rightarrow$ $(X, A)$ by defining

$$
\xi: A(X) \rightarrow M^{\infty}\left(\mathbf{Z}_{2} \operatorname{Alg}\left(A X, \Lambda \mathbf{R}^{L}\right), \Lambda \mathbf{R}^{L}\right)
$$

by

$$
\xi(a)(y)=y(a) \text { for all } y \text { in } \mathbf{Z}_{2} \operatorname{Alg}\left(A X, \Lambda \mathbf{R}^{L}\right) .
$$

It must be shown that $\xi(a)$ is smooth. This is a "local" result, and it is enough to show that for $a$ in $C^{\infty}(V) \otimes \Lambda \mathbf{R}^{s}$ for some open set $V$ in $\mathbf{R}^{r}$,

$$
\xi(a): \mathbf{Z}_{2} \operatorname{Alg}\left(C^{\infty}(V) \otimes \Lambda \mathbf{R}^{s}, \Lambda \mathbf{R}^{L}\right) \rightarrow \Lambda \mathbf{R}^{L}
$$

is smooth. This follows by observing that $\xi(a)=\psi^{-1} \circ Q$, where $Q$ is the bijection of Lemma 3.2 and $\psi$ is the isomorphism of $2.7(\mathrm{v})$.

By general properties of sheaves, we can choose an open cover $\left\{U_{i}\right\}$ of $X$ and write $A X$ in terms of the algebras $A\left(U_{i}\right)$,

$$
A\left(U_{i}\right)=\left\{\left(a_{i}\right) \in \prod_{i} A\left(U_{i}\right): a_{i}=a_{j} \text { when restricted to } A\left(U_{i} \cap U_{j}\right) \text { for all } i, j\right\}
$$


That $\boldsymbol{\xi}$ is an algebra homomorphism then also depends on "local" properties. If $V \subset W$ are open sets in $\mathbf{R}^{r}$, it is enough to show that $\xi$ induces isomorphisms which make the following diagrams commute.

$$
\begin{array}{ccc}
C^{\infty}(W) \otimes \Lambda \mathbf{R}^{s} & \stackrel{\xi_{W}}{\rightarrow} & M^{\infty}\left(\mathbf{Z}_{2} \operatorname{Alg}\left(C^{\infty}(W) \otimes \Lambda \mathbf{R}^{s}, \Lambda \mathbf{R}^{L}\right), \Lambda \mathbf{R}^{L}\right) \\
\text { restriction } \downarrow & & \downarrow \text { restriction } \\
C^{\infty}(V) \otimes \Lambda \mathbf{R}^{s} & \stackrel{\xi_{V}}{\rightarrow} & M^{\infty}\left(\mathbf{Z}_{2} \operatorname{Alg}\left(C^{\infty}(V) \otimes \Lambda \mathbf{R}^{s}, \Lambda \mathbf{R}^{L}\right), \Lambda \mathbf{R}^{L}\right)
\end{array}
$$

Using $Q$ to identify $\left.\mathbf{Z}_{2} \operatorname{Alg}\left(C^{\infty}(W) \otimes \Lambda \mathbf{R}^{s}, \Lambda \mathbf{R}^{L}\right)\right)$ with $\varepsilon^{-1}(W) \subset E^{r, s}, \xi_{W}$ is seen to be $\psi^{-1}$ of $2.7(v)$. Similarly $\eta_{V}$ is an isomorphism and the diagram is easily shown to be commutative.

Define $\eta:(X, A) \rightarrow M_{L} H_{L}(X, A)$ to be $\xi^{-1}$.

3.8. Definition at $\zeta$. Let $S$ be a supermanifold. Define

$$
\zeta: S \rightarrow Z_{2} \operatorname{Alg}\left(M^{\infty}\left(S, \Lambda \mathbf{R}^{L}\right), \Lambda \mathbf{R}^{L}\right)=H_{L} M_{L}(S)
$$

by

$$
\zeta(s)(f)=f(s) \text { for all } f \text { in } M^{\infty}\left(S, \Lambda \mathbf{R}^{L}\right) \text {. }
$$

The map $\zeta(s)$ is an algebra homomorphism since the map $I$ of 2.5(iv) is an algebra homomorphism.

That $\zeta$ is a superdiffeomorphism follows from the fact that $\zeta$ restricts to superdiffeomorphisms on charts. It is enough to show for an open set $U$ in $E^{r, s}$,

$$
\zeta: U \rightarrow \mathbf{Z}_{2} \operatorname{Alg}\left(M^{\infty}\left(U, \Lambda \mathbf{R}^{L}\right), \Lambda \mathbf{R}^{L}\right)
$$

is a superdiffeomorphism. By $2.7(v), \psi: M^{\infty}\left(U, \Lambda \mathbf{R}^{L}\right) \rightarrow C^{\infty}\left(\varepsilon(U) \otimes \Lambda\left(\pi_{j}\right)\right)$ is an isomorphism. Then $\zeta$ can be written as the composition of two maps,

$$
\begin{aligned}
H_{L}(\psi) \circ Q^{-1}: U & \rightarrow \mathbf{Z}_{2} \operatorname{Alg}\left(C^{\infty}(\varepsilon(U)) \otimes \Lambda \mathbf{R}^{s}, \Lambda \mathbf{R}^{L}\right) \\
& \rightarrow \mathbf{Z}_{2} \operatorname{Alg}\left(M^{\infty}\left(U, \Lambda \mathbf{R}^{L}\right), \Lambda \mathbf{R}^{L}\right),
\end{aligned}
$$

where $Q$ is the bijection of Lemma 3.2. The map $Q^{-1}$ defines the smooth structure on $Z_{2} \operatorname{Alg}\left(C^{\infty}\left(\varepsilon(U) \otimes \Lambda \mathbf{R}^{s}\right), \Lambda \mathbf{R}^{L}\right)$, and $H_{L}(\psi)$ is a smooth map by Lemma 3.4. Since $H_{L}$ is a functor, the fact that $\psi$ is an isomorphism implies that $\zeta$ is a superdiffeomorphism.

The proof is completed by observing that the following diagrams are commutative, for any morphism $\sigma:(X, A) \rightarrow\left(X^{\prime}, A^{\prime}\right)$ of graded manifolds or $\tau: S \rightarrow S^{\prime}$ of supermanifolds.

$$
\begin{array}{cccccc}
(X, A) & \rightarrow & M_{L} H_{L}(X, A) & S & \rightarrow & H_{L} M_{L}(S) \\
\downarrow \sigma & & \downarrow M_{L} H_{L}(\sigma) & \downarrow \tau & & \downarrow H_{L} M_{L}(\tau) \\
\left(X^{\prime}, A^{\prime}\right) & \rightarrow & M_{L} H_{L}\left(X^{\prime}, A^{\prime}\right) & S^{\prime} & \rightarrow & H_{L} M_{L}(S)
\end{array}
$$

3.9. Proof of Proposition 1.10. Part (ii) is immediate. For part (i), write $E^{r, s}(L)$ and $E^{r, s}\left(L^{\prime}\right)$ in order to distinguish $E^{r, s}$ defined via $\Lambda \mathbf{R}^{L^{\prime}}$. Similarly write $\varepsilon_{L}$ and $\varepsilon_{L^{\prime}}$ to distinguish between the corresponding augmentation maps. Let $S$ be a supermanifold in $\mathcal{S} \Re_{L}$ and let $a=\left\{\left(U_{\alpha}, \phi_{\alpha}\right)\right\}$ be the maximal atlas which defines $S$. 
Then define

$$
\bar{S}=\bigcup_{\alpha} \varepsilon_{L^{\prime}}^{-1} \varepsilon_{L}\left(\phi_{\alpha}\left(U_{\alpha}\right)\right) / \sim,
$$

where the equivalence relation $\sim$ is defined as follows. Notice that if $U$ is an open set in $E^{r, s}(L)$,

$$
M^{\infty}\left(U, \Lambda \mathbf{R}^{L}\right) \cong C^{\infty}\left(\varepsilon_{L}(U) \otimes \Lambda \mathbf{R}^{s}\right) \simeq M^{\infty}\left(\varepsilon_{L^{\prime}}^{-1} \varepsilon_{L}(U), \Lambda \mathbf{R}^{L^{\prime}}\right)
$$

Then the superdiffeomorphisms

$$
\phi_{\alpha} \phi_{\beta}^{-1}: \phi_{\beta}\left(U_{\alpha} \cap U_{\beta}\right) \rightarrow \phi_{\alpha}\left(U_{\alpha} \cap U_{\beta}\right)
$$

give rise to superdiffeomorphisms

$$
\tau_{\alpha \beta}: \varepsilon_{L^{\prime}}^{-1} \varepsilon_{L} \phi_{\beta}\left(U_{\alpha} \cap U_{\beta}\right) \rightarrow \varepsilon_{L^{\prime}}^{-1} \varepsilon_{L} \phi_{\alpha}\left(U_{\alpha} \cap U_{\beta}\right) \text {. }
$$

Then if $a$ is in $\varepsilon_{L^{\prime}}^{-1} \varepsilon_{L} \phi_{\alpha}\left(U_{\alpha}\right)$ and if $b$ is in $\varepsilon_{L^{\prime}}^{-1} \varepsilon_{L} \phi_{\beta}\left(U_{\beta}\right)$, then $a \sim b$ if $\tau_{\alpha \beta} b=a$.

It is not hard to check that $\tilde{S}$ is in $\delta \mathscr{N}_{L^{\prime}}$. That $\delta \mathscr{R}_{L}$ includes as a full subcategory in $\mathcal{S} \Re_{L^{\prime}}$ also follows from (B).

\section{REFERENCES}

1. M. Batchelor, The structure of supermanifolds, Trans. Amer. Math. Soc. 253 (1979), 329-338.

2. F. Berezin, The method of second quantization, Academic Press, New York, 1966.

3. F. Berezin and G. Kac, Lie groups with commuting and anticommuting parameters, Math. USSR-Sb. 11 (1970), 311-326.

4. F. Berezin and D. Leites, Supervarieties, Soviet Math. Dokl. 16 (1975), 1218-1222.

5. L. Corwin, Y. Ne'eman and S. Sternberg, Graded Lie algebras in mathematics and physics (Base-Fermi symmetry), Rev. Modern Phys. 47 (1975), 573-604.

6. B. de Witt, Differential supergeometry (in preparation).

7. V. Kac, Lie superalgebras, Advances in Math. 26 (1977), 8-96.

8. B. Kostant, Graded manifolds, graded Lie theory and prequantization, Differential Geometric Methods in Mathematical Physics, Lecture Notes in Math., vol. 570, Springer-Verlag, Berlin and New York, 1977, pp. 177-306.

9. W. Rudin, Functional analysis, McGraw-Hill, New York, 1973.

10. M. Sweedler, Hopf algebras, Benjamin, New York, 1969.

11. F. Treves, Topological vector spaces, distributions and kernels, Academic Press, New York, 1967.

Department of Pure Mathematics and Mathematical Statistics, University of Cambridge, 16 Mill lane, Cambridge CB2 1SB, England 